\title{
Weak Central Coherence Contributes to Social Perceptual Deficits in autism
}

\author{
M.D Rutherford", Nidhi Trivedi, Patrick J. Bennett and Allison B. Sekuler
}

*Correspondence: rutherm@mcmaster.ca

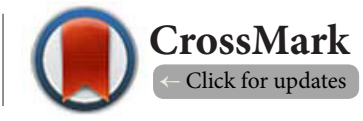

Department of Psychology, Neuroscience and Behaviour, McMaster University, Hamilton, Ontario, Canada.

\begin{abstract}
The weak central coherence theory of autism originally suggested that people on the autism spectrum focus on features rather than perceiving a global gestalt. To test whether central coherence theory is relevant to social perception in autism, we tested whether global processing on a non-social task was associated with global processing on a social task. Fourteen adults with autism and 14 matched controls completed a Navon task and an emotion perception task with a point light-walker display. Across both groups as well as within the autism group, there was a correlation between global processing in the Navon task and the social task. This association that global processing may underpin performance on both tasks.
\end{abstract}

Keywords: Autism Spectrum Disorders, Central Coherence Theory, Navon Task, Social Perception

\section{Introduction}

Successful visual perception relies on perceiving both local and global order [30]. There is a consensus that there are group differences in perception when those with and without autism spectrum disorders (ASD) are compared, including a stronger tendency on the part of neurotypical observers to integrate a visual scene into a meaningful whole (global processing), or a stronger tendency on the part of those on the autism spectrum to perceive features and individual parts (local processing; for reviews, see $[5,57]$. For example, group-wise comparisons have shown that people on the autism spectrum have superior performance in the visual perception of hidden forms such as finding a target triangle in a line-drawing of a baby's stroller [55] and show superior performance on visual search tasks in which a small target stimulus (e.g. the letter S) must be located while it is displayed in an array of other letters $[43,46]$. Global perception is best in a group on the autism spectrum when stimulus presentation times are long, but best in control groups when stimulus presentation times are short [65] The current experiment tested whether a tendency of people on the autism spectrum to see features instead of a coherent whole is related to social perception, as originally predicted by Weak Central Coherence theory [19].

\section{Global precedence in visual processing} A hallmark of human visual processing is the tendency to see a meaningful whole when viewing a visual scene $[67,40]$. Experimentally, this holistic processing is evident when features cannot be processed without interference from other parts of the image [69] or when global information is processed faster than local information [41]. In the classic Navon task, participants are briefly presented with a large letter that is composed of smaller letters. The smaller and larger letters might be the same (congruent trials) or different (incongruent trials). Navon reported interference introduced only by larger (global), not smaller (local) letters. In other words, the identity of larger letters affected perception of smaller letters only [40].

The weak central coherence theory of visual processing in ASD

Proponents of the Weak Central Coherence (WCC) theory of ASD originally suggested that instead of spontaneously integrating features of a scene into a meaningful whole, as neurotypical observers would, those on the autism spectrum tend to perceive the unintegrated features $[13,14,19,21,22,23]$ suggested that people on the autism spectrum show"detail-focused processing in which features are perceived and retained at the expense of global configuration and contextualized meaning" [21], p. 217). Past research has found group differences in performance on the Navon task when groups with and without autism are compared [47]. Enhanced local processing may underlie superior performance among participants on the autism spectrum 
Rutherford et al., Journal of Autism 2020,

on some tasks such as the Block Design [56] and Embedded Figures tasks $[\mathbf{2 8}, \mathbf{4 2}, \mathbf{5 5}]$. Used a multiple object tracking paradigm to discover a reduced bias towards global processing in a group with ASD compared to a control group. Recently [8] reported evidence that compared to matched controls, their sample on the autism spectrum were less sensitive to global geometric impossibility, and concluded that weak global integration was characteristic of autistic perception. A recent meta-analysis including various methods for measuring local and global processing report a moderate decrement in global processing (Hedges' $g=-0.47$ ) when participants with ASD were compared to controls, but no group differences in local processing. This group difference was large when the tasks involve local processing as interference with the required global processing (Hedges' $g=-0.98 ; 64$ ).

That said, the local processing that is characteristic of autism may vary with task type and instructions e.g. [38,64], and [38] suggested that focus on global or local information maybe under volitional control. Furthermore, it has been suggested that local and global processing are independent, rather than mutually exclusive, and that local and global processing rely on different psychological mechanisms $[22,36,63]$. In support of this idea, a recent study using a face perception task reported that although a local processing bias may be characteristic of autistic perception, this bias is not accompanied by a decrement in global perception $[18,60]$. Reported a featural processing bias and unimpaired global processing ability in ASD, and Almeida et al., (2014) reported enhanced local processing along with superior contour integration in a sample with ASD. A recent meta-analysis suggests that the most robust measure of group differences in local versus global processing is reaction time, and that people on the autism spectrum are slower to complete tasks that require global processing [64].

\section{Weak Central Coherence and Social Perception}

There is robust evidence of autism-specific visual processing of social information, including differences in face perception $[15,29,32]$, and in perceptual strategies used while viewing emotional facial expressions $[39,50,51]$. Some evidence suggests less spontaneous perception of animate motion among a sample of children with ASD compared to neurotypical controls [52]. People on the autism spectrum may perceive the biological motion depicted by point light walkers less spontaneously than neurotypical controls [31]. Observers on the autism spectrum have been shown to be less accurate as a group than neurotypical controls when detecting the presence of a human form amid masking dots [7] and in reporting the left or right direction of motion of a point light walker. This is especially true when the task involves the perception of emotions in a point light display $[2,26,44]$.

An association between Weak Central Coherence and Theory of Mind is also posited by Skorich and colleagues $[6,58,59]$ who suggest that Weak Central Coherence affects the cogni- tive process of self-categorization, which in turn disrupts the shared attention mechanism, one of the components of the theory of mind system. Their evidence for this is an association among neurotypical participants between scores on the autism-spectrum quotient and theory of mind performance, mediated by a social categorization task. They also report that those with higher AQ scores showed less in group favoritisms in a minimal group paradigm as a function of decreased selfcategorization $[6,19]$. Originally suggested that the social cognitive anomalies of autism can ultimately be explained by the tendency to favor local over global information during visual processing, but [23] subsequently proposed some modifications of the original framing, specifically suggesting that the weak central coherence was independent of social cognitive performance in autism.

Fundamental to the current study is the question of whether central coherence is related to $[19,58,59]$, or independent of [23], social perception in autism. By measuring the relationship between social and non-social measures of global precedence, we can test whether social perceptual anomalies in autism are related to central coherence.

\section{The current study}

The weak central coherence theory proposes a broad explanation for perceptual differences that is meant to encompass social and non-social domains of perception. Whether group differences in global processing explain group differences in social perception remains an open question. We reasoned that if weak central coherence drives the visual processing differences in autism, then measures of global (or local) biases in visual processing in one task should be associated with such processing in another task. This should be true even across domains: there should be an association between the tendency to process information globally on a social task and the tendency to process information globally on a non-social task.

In this study, we assessed a group of young adults on the autism spectrum and a matched control group using a Navon task (a non-social task) and a point light walker task (a social task). The weak central coherence theory predicts a correlation between global biases on the non-social task and accuracy on the social perception task in the ASD group. Such a correlation is expected to be weaker in the control group, as specialized social perceptual processes are expected to be employed in the social perception task. If visual perceptual processes that are specialized for social perception are used in the PLW task e.g. [16,17], these same perceptual processes would not be recruited for performance in the Navon task, and such processing would be independent of a tendency toward global precedence. Therefore, we tested two predictions: 1) there is a significant relationship between Global Precedence on the Navon task and Global Precedent on the point-light walker task; and 2) this relationship between Global Precedence across the two tasks is smaller for the control group than for the ASD group. 


\section{Methods}

\section{Participants}

There were 28 adult male participants. [63] Found a correlation of .81 between attention to detail and the social problems subscale of the Social Responsiveness Scale [11]. Based on that study, which is the only study we know of that compares global processing and social skills, a sample of 9 participants should be sufficient for a robust estimate of correlation [27].

There were 14 participants in the autism group (age range $=21.5$ years to 30.4 years), recruited via referral from a clinical specialist treating autism spectrum disorders. Each had been diagnosed with Autism Spectrum Disorder by a clinician, and this diagnosis was confirmed by one of the authors (MDR) for this study using: (1) the Autism Diagnostic Observation Schedule (ADOS-G; 33) and (2) the Autism Diagnostic Interview (ADI-R; 34). MDR has been trained to research reliability for ADOS-G and ADI-R administration. There were 14 participants in the control group (age range $=25.5$ years to 34 years), recruited from the community through an online advertisement. Participants in both groups had normal or corrected-to-normal visual acuity which was checked prior to participation using a Snellen eye chart. Participants were free from any other medical, psychiatric or developmental disorders. IQ estimates were obtained using the Wechsler Adult Intelligence Scale (WAIS-IV; 66). Because of the non-verbal nature of the task, the groups were matched on PIQ $(t(26)=0.19 . p=0.85)$. Each group's demographic information is shown in Table 1.

Table 1. Demographic information for all participants.

\begin{tabular}{llllll}
\hline & Age & FSIQ & VIQ & PIQ & $\begin{array}{l}\text { Total } \\
\text { ADOS } \\
\text { score }\end{array}$ \\
\hline ASD & $26.5(3.6)$ & $97.6(9.5)$ & $99.5(13.6)$ & $97.5(10.5)$ & $12.8(4.9)$ \\
Control & $28.8(5.7)$ & $103.3(13.7)$ & $107.6(10.6)$ & $98.5(16.1)$ & N/A \\
& $t=1.28$ & $t=1.28$ & $t=1.88$ & $t=0.19$ & -- \\
& $p=0.21$ & $p=0.22$ & $p=0.08$ & $p=0.85$ & \\
\hline
\end{tabular}

\section{Compliance with Ethical Standards}

This research involving human participants was carried out in accordance with the Code of Ethics of the World Medical Association (Declaration of Helsinki). Informed written consent was obtained, and research was approved by the University Research Ethics Board. The authors had no conflicts of interest.

\section{Apparatus}

A computer with the Video Toolbox [45] and Psychophysics Toolbox [9] extensions for MATLAB was used to present the stimuli. Stimuli were presented on a 19-inch CRT that had a resolution of $1024 \times 760$ pixels and a refresh rate of $85 \mathrm{~Hz}$.

\section{Stimuli: Point light walkers}

Point-light walker stimuli were based on Cutting's classic point light walker algorithm [10]. The walker was made up of 11 point-lights that represented the head, one shoulder, both elbows, both wrists, one hip, both knees, and both ankles. These 11 dots moved coherently to present a walker moving to the left or right at a speed of 7 degrees per second. Walkers subtended $1.9^{\circ} \times 4.2^{\circ}$. All walkers were presented for 1.128 seconds at a frame rate of $25 \mathrm{fps}$, such that a stride cycle was completed after 80 frames. Dot luminance was $67.4 \mathrm{~cd} /$ $\mathrm{m}^{2}$ and the background luminance was less than $1 \mathrm{~cd} / \mathrm{m}^{2}$. Half of the walkers moved as if happy and half moved as if angry, each emotion created using gaits of people walking either happily or angrily (for more details, see 49). The point-light walker remained centered on the screen as it walked, as if it were on a treadmill, rather than translating across the screen. The dynamic noise mask comprised dots that were identical in size and contrast to those that made up the walker.

Four conditions were created: 1) Upright; 2) Inverted; 3) Scrambled; and 4) Mixed. In the Inverted condition, the original walker was rotated 180 degrees in the fronto-parallel plane so that it appeared to be walking upside-down on a ceiling. This condition preserves (thus controls for) both local and global visual information and motion, while interfering with social perception.

In the Scrambled condition, the starting vertical position of each dot was randomly selected along the vertical axis of the walker, but its motion trajectory was preserved. Thus, the local motion of each dot, but not the walker's global form, was preserved. While the direction of intact point-light displays could be discerned using either global or local information [61], in a scrambled walker, global information was unavailable. Therefore, accurately discriminating left versus right movement of the scrambled walker is evidence that the observer used local motion to discern the direction. It is likely the observers rely on the dot representing the foot, which still appears to lift, plant, and traverse a horizontal plane [61].

In the Mixed condition, each dot was shifted randomly between two adjacent joints across successive frames. This manipulation disrupted local trajectories of individual dots but preserved the walker's global form. Hence, accurate discrimination of the point light walker's direction of motion would imply that the observer is sensitive to global form information. Together, these four conditions allow us to isolate the effects of local and global information on the discrimination of point light walkers.

\section{Stimuli: Navon task}

The stimuli in the Navon tasks were composed of larger digits $(1.8 \times 2.6 \mathrm{~cm})$ created by arrangements of smaller digits $(0.2 \times 0.3$ $\mathrm{cm})$, modelled after the traditional Navon tasks [40]. In congruent stimuli, the global numeral was made up of smaller numerals that were the same number (e.g. both " 3 " or both "6"). In incongruent stimuli, the global numeral (e.g. "6") was made up of smaller numerals that were different (e.g. "3s"). In neutral stimuli, the numbers 3 or 6 occurred at either the local or global level, but not both. Thus, neutral stimuli consisted 
Rutherford et al., Journal of Autism 2020,

http://www.hoajonline.com/journals/pdf/2054-992X-7-2.pdf

doi: $10.7243 / 2054-992 X-7-2$

of local $3 \mathrm{~s}$ or $6 \mathrm{~s}$ were arranged to form global $2,5,8$, or $9 \mathrm{~s}$, or global $3 \mathrm{~s}$ or $6 \mathrm{~s}$ made up of local $2,5,8$, or $9 \mathrm{~s}$.

\section{Procedure}

Each observer viewed the stimuli binocularly from a distance of $100 \mathrm{~cm}$. Viewing position was stabilized with a chin and forehead rest that aligned the participants' eyes with the center of the computer screen. The display was the only source of light in the testing room. Each participant completed the Point Light Walker Task followed by the Navon Task.

\section{Point Light Walker Tasks}

Participants completed a "Direction" task and an "Emotion" task with point light walker stimuli. In the Direction task, participants decided whether a point light walker was moving to the left or to the right by pressing a labelled key on a standard computer keyboard. Participants were asked to respond accurately and quickly. Six familiarization trials of each walker type (12 total trials) were presented prior to the test trials. Auditory feedback was provided during the familiarization trials but not for experimental trials. Each participant performed 96 trials for each of the 4 stimulus conditions, for a total of 384 trials. Stimuli were visible until response. Trial types were presented in a randomized order.

In the Emotion task, participants had to decide whether the point light walker was happy or angry by pressing keys on keyboard that were labelled "happy" or "angry". As in the Direction task, participants were asked to respond accurately and quickly. Six point-light displays of each type were presented to the participants in practice trials. Auditory feedback was provided on familiarization trials but not experimental trials. Each participant performed 96 trials for each of the 4 stimulus conditions, for a total of 384 trials. Stimuli were visible until response. Trial types were presented in a randomized order.

\section{Navon task}

In all Navon tasks, the participants were asked to determine if the stimulus contained a " 3 " or a " 6 " and to respond quickly and accurately by pressing a key on a computer keyboard. The stimuli were present until the participant responded, and accuracy and reaction time were recorded. In the local task, the participants were presented 144 trials (48 neutral, 48 congruent, 48 incongruent, presented in a randomized order), and were asked to base their decision on the local/ small numerals. In the global task, the participants were presented 144 trials (48 neutral, 48 congruent, 48 incongruent, presented in a randomized order), and were asked to base their decision on the global/large numerals. The local and global tasks were presented in separate blocks of trials. Half of the participants completed the local block first, and half completed the global block first. There were no practice trials.

\section{Statistical Procedures}

Statistical analyses were performed in R (R Core Team, 2018).
Where appropriate, the Huynh-Feldt correction was used to adjust the degrees-of-freedom and $p$ values to correct for violations of the sphericity assumption underlying $\mathrm{F}$ tests for within-subject variables [35]. The association strength between two variables was expressed as partial-eta-squared $\left(\eta_{\mathrm{p}}{ }^{2}\right)$. Two-sample $t$ tests used degrees-of-freedom that were adjusted using the Welch-Satterthwaite correction for unequal variances, and effect size was expressed as Cohen's $d$.

Our analyses are as follows: First, we compared response accuracy across groups on the Navon and PLW tasks in order to ensure that they were comparable. Next, we examined group differences on reaction time in the Navon and PLW tasks. Reaction times were log transformed to normalize the distributions for analyses. Finally, we evaluated group differences on indices of Global and Local Interference that were based on reaction times in the Navon and PLW tasks. For example, the index of Global Interference in the global condition on the Navon task was calculated by computing the log difference between correct reaction times to congruent and incongruent stimuli in the Global task. Finally, and most significantly, we evaluated the correlation between Global and Local Interference on the Navon and PLW tasks. A correlation would be predicted by the Weak Central Coherence Theory and would lend support to the idea that a Weak Central Coherence explains the lower Global Interference (greater local interference) on both tasks in the autism group.

\section{Global \& Local Interference in the Navon Task}

A single measure of Global Interference in the Navon task was created by averaging two congruency effects based on reaction times from trials in the Global condition in which responses were correct. Distributions of reaction times typically are positively skewed and have a variance that grows with the mean. Therefore, our measure of Global Interference was based on log-transformed reaction times to normalize the data and reduce differences in variance across conditions. The first congruency effect was defined as the difference between log-transformed reaction times on Local-Incongruent trials, in which observers identified the smaller numerals that comprised a different larger numeral, and Local-Congruent trials, in which observers identified the smaller numerals that comprised the same larger numeral. The second congruency effect was defined as the difference between log-transformed reaction times on Local-Neutral trials, in which observers identified the smaller numerals that comprised a different larger numeral and Local-Congruent trials. To the extent that participants can identify the local elements and ignore the global structure, reaction times on Congruent, Incongruent, and Neutral trials should be similar and the congruency effects should be approximately zero. To the extent that the global numeral cannot be ignored, reaction times should be lower on congruent trials than incongruent and neutral trials, and therefore the congruency effects should be greater than zero. The two congruency effects were correlated across our 
Rutherford et al., Journal of Autism 2020,

participants $(r=0.67, p<.01)$, and therefore they were averaged to create a single measure of Global Interference for the Navon task. In summary, our index of Global Interference was:

Log10(RT Local-Incongruent / RT Local-Congruent) + Log10(RT Local-Neutral / RT Local-Congruent)

We also computed an index of Local Interference by averaging two congruency effects based on correct reaction times from trials in the Local condition. The first congruency effect was defined as the difference between log-transformed reaction times on Global-Incongruent and Global-Congruent trials. The second congruency effect was defined as the difference between log-transformed reaction times on Global-Neutral and Global-Congruent trials. To the extent that participants can identify the global structure and ignore the local elements, reaction times on Congruent, Incongruent, and Neutral trials should be similar and the congruency effects should be approximately zero. To the extent that the local elements cannot be ignored, reaction times should be lower on congruent trials than incongruent and neutral trials, and therefore the congruency effects should be greater than zero. The two congruency effects were correlated across our participants $(r=0.70, p<.01)$, and therefore they were averaged to create a single measure of Global Interference for the Navon task. In summary, our index of Global Interference was:

Log10(RT Global-Incongruent / RT Global-Congruent) + Log10(RT Global-Neutral / RT Global-Congruent)

\section{Global Precedence in the Point Light Walker Task}

Separate measures of Global Precedence were created for the Direction and Emotion tasks; each one was created by averaging two measures of Global Precedence. If participants relied on global information to perform the PLW tasks, then we expected reaction time to be shorter in the Upright and Mixed conditions, in which global information is preserved, than in the Scrambled and Inverted conditions, in which global information was missing or presented at an unfamiliar orientation. Therefore, we computed the log-difference between i) the average reaction time in the Inverted and Scrambled conditions; and ii) the average reaction time in the Upright and Mixed conditions.

Finally, we created a measure of Global Precedence based on response accuracy. To the extent that participants rely on global structure to discriminate $\mathrm{PL}$, we expected accuracy to be higher in the Upright and Mixed conditions than in the Inverted and Scrambled conditions. Therefore, we computed the difference between i) the average of the proportion of correct responses in the upright and mixed conditions; and ii) the average of accuracy in the inverted and scrambled conditions. There were obvious ceiling effects on response accuracy in the PLW-Direction task, and therefore we computed this measure of Global Precedence only using data from the PLW-Emotion task.

\section{Results}

Our analyses included a test of our two primary hypotheses:

1) That there would be a correlation between Global Precedence on the Navon task and Global Precedent on the PLW task; and 2) this relationship between Global Precedence across the two tasks is smaller for the control group than for the ASD group. Prior to conducting these analyses, we first compared accuracy and reaction time across the two groups in both the Navon task and the PLW task in order to ensure that the distributions were comparable. We then tested for evidence of Global Precedence and Local interference using congruency effects. Next, we computed an index of Global Precedence for both the Navon and the PLW task so that we could test the two main hypotheses.

\section{Accuracy \& Reaction Time in the Navon Task}

The proportion of errors was less than $5 \%$ in both groups and all conditions. Across all conditions, the average percent correct was 98.4 and 98.7 in the autism and Control groups, respectively. Within the ASD group, there was a small negative correlation between accuracy and Social Affect (SA) ADOS scores that did not reach significance $(r=-0.28, p=0.33)$. Hence, response accuracy was high and there was no indication that it varied between groups or conditions. Because response accuracy was so high, the data were highly non-normal due to ceiling effects, and therefore we did not analyze response accuracy further.

The distributions of reaction times in the Navon tasks are illustrated in Figure 1. To a first approximation, the distributions of log reaction time were symmetrical about the median and the spread of the data was similar across conditions. Furthermore, in all conditions the median reaction time was higher in the autism group than the neuro-typical group. Within the ASD group, there was a moderate positive correlation between reaction times and Social Affect (SA) ADOS scores that did not reach significance $(r=0.41, p=0.14)$. Log-transformed reaction times were analyzed with a 2 (group: ASD vs Control) x 2 (task: Local vs. Global) x 3 (condition: Congruent, Incongruent, Neutral) ANOVA. The ANOVA found significant main effects of group $\left(F(1,26)=8.63, \eta_{\mathrm{p}}{ }^{2}=0.25, p=0.007\right)$, task $\left(F(1,26)=8.99, \eta_{\mathrm{p}}{ }^{2}=0.26\right.$, $p=0.006)$, and condition $\left(F(2,52)=51.34, \eta_{p}{ }^{2}=0.66, p<0.001\right)$. These main effects were qualified by a significant task $x$ condition interaction $\left(F(1.87,48.62)=6.52, \eta_{p}{ }^{2}=0.20, p=0.003\right)$ and a significant three-way interaction between group, task, and condition $\left(F(1.87,48.62)=4.02, \eta_{p}{ }^{2}=0.13, p=0.02\right)$. The three-way interaction was analyzed by conducting separate 2 (group) $\times 3$ (condition) ANOVAs on data from the local and global tasks. In the local task, the main effects of group $(F(1,26)=4.47$, $\left.\eta_{\mathrm{p}}{ }^{2}=0.15, p=0.04\right)$ and condition $\left(F(1.93,50.18)=59.89, \eta_{\mathrm{p}}{ }^{2}=0.70\right.$, $p<0.001)$ were significant, but the group $x$ condition was not $\left(F(1.93,50.18)=0.21, \eta_{p}{ }^{2}=0.008, p=0.81\right)$. In the global task, the main effects of group $\left(F(1,26)=8.69, \eta_{p}{ }^{2}=0.25, p=0.007\right)$ and 
Rutherford et al., Journal of Autism 2020,

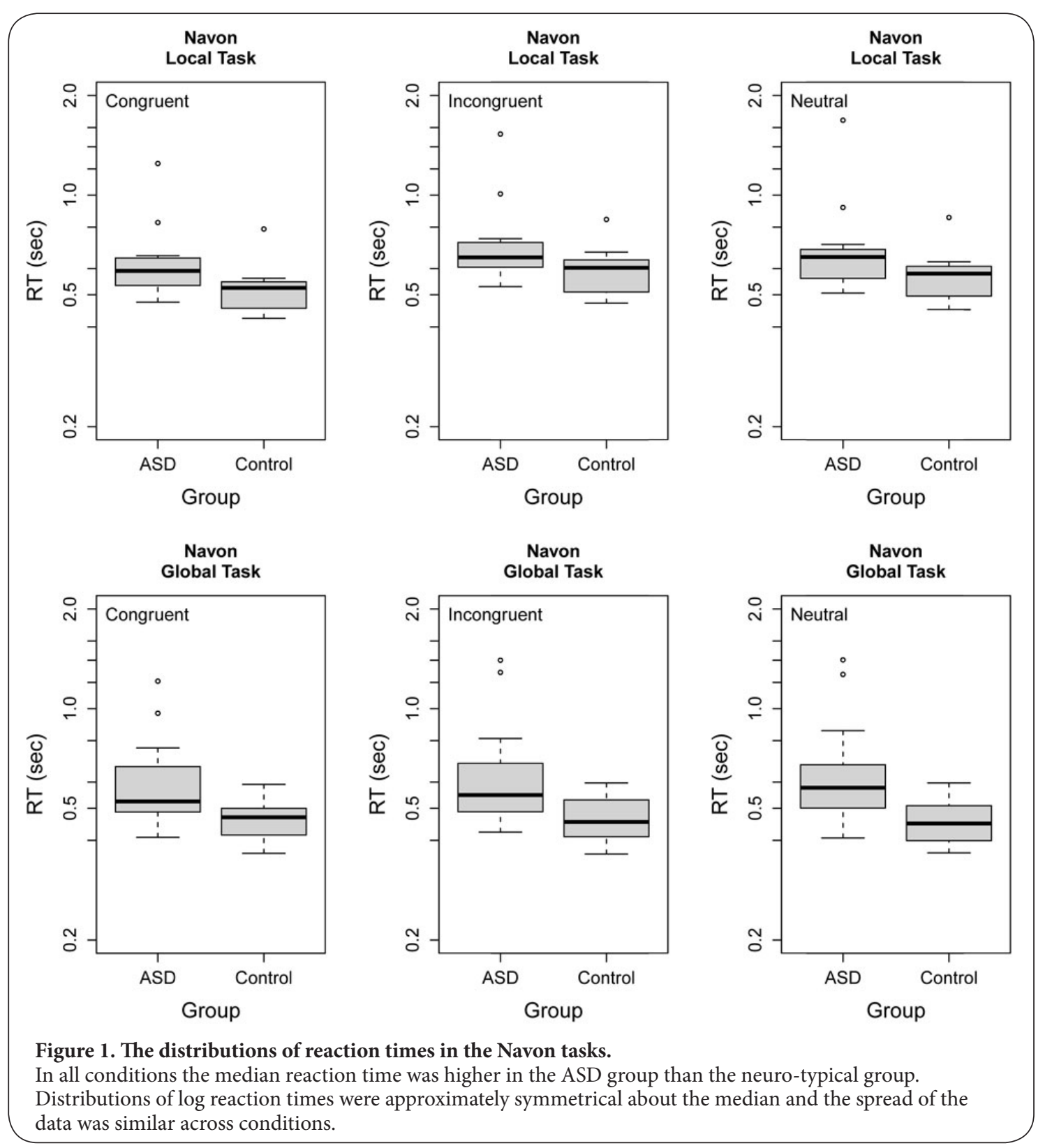

condition $\left(F(2,52)=6.76, \eta_{\mathrm{p}}^{2}=0.21, p=0.002\right)$ and the group $\mathrm{x}$ condition interaction $\left(F(2,52)=7.06, \eta_{p}^{2}=0.21, p=0.002\right)$ were significant. The interaction was attributable to the fact that the group difference in the neutral condition $(F(1,26)=10.16$, $\left.\eta_{\mathrm{p}}{ }^{2}=0.28, p=0.004\right)$ was larger than the group difference in the congruent $\left(F(1,26)=7.77, \eta_{\mathrm{p}}{ }^{2}=0.23, p=0.01\right)$ and incongruent $\left(F(1,26)=7.83, \eta_{\mathrm{p}}{ }^{2}=0.23, p=0.01\right)$ conditions, although this difference is modest.

In summary, accuracy was very high in all conditions in both groups. In addition, reaction times on correct trials were longer in participants on the spectrum than Control participants, and this group difference was slightly greater in the Global task than the Local task.
Accuracy \& Reaction Time in the Point Light Walker Task The proportions of errors in the Point Light Walker tasks were much higher than those found in the Navon tasks. In the PLW-Direction discrimination task, the error rates were similar in the two groups but varied noticeably across conditions: in both groups, error rates were lower than $5 \%$ in the Upright and Mixed conditions, approximately 14\% in the Inverted condition, and 39\% in the Scrambled condition. In the PLW-Emotion discrimination task, error rates were 5-10\% higher in the autism group than the Control group: the average error rates in the autism group were $25 \%$ in the Upright condition, 39\% in the Inverted condition, 44\% in Scrambled condition, and $27 \%$ in the Mixed condition. Within the ASD 
group, there was a moderate negative correlation between accuracy and Social Affect (SA) ADOS scores that did not reach significance $(r=0-.44, p=0.11)$. The distributions of response accuracy were highly non-normal in the Upright and Mixed conditions in the Direction discrimination task, and therefore we did not submit all of the accuracy to an ANOVA. Instead we used separate ANOVAs to analyze all of the conditions in the Emotion task, and the Inverted and Scrambled conditions in the Direction task. In the Emotion task, the main effects of group $\left(F(1,26)=5.53, \eta_{\mathrm{p}}{ }^{2}=0.18, p=0.03\right)$ and condition $\left(F(2.01,52.37)=26.77, \eta_{\mathrm{p}}{ }^{2}=0.51, p<0.001\right)$ were significant, but the group $\mathrm{x}$ condition interaction was not $\left(F(2.01,52.37)=0.25, \eta_{\mathrm{p}}{ }^{2}=0.01, p=0.86\right)$. In the Direction task, the main effect of condition was significant $(F(1,26)=34.48$, $\left.\eta_{\mathrm{p}}{ }^{2}=0.57, p<0.001\right)$, but the main effect of group $(F(1,26)=0.01$, $\left.\eta_{\mathrm{p}}^{2}<0.001, p=0.91\right)$ and the two-way interaction $(F(1,26)=0.01$, $\left.\eta_{\mathrm{p}}{ }^{2}<0.001, p=0.91\right)$ were not. Hence, error rates in the PLW tasks were much higher than in the Navon tasks and varied significantly across conditions. Also, we found that error rates were higher (i.e., accuracy was lower) in the ASD group than the Control group in the Emotion discrimination task but not the Direction discrimination task.

The distributions of reaction times in the PLW tasks are illustrated in Figure 2. As was the case in the Navon tasks, the distributions of log reaction time were approximately symmetrical about the median and the spread of the data was similar across conditions. Unlike what was found in the Navon tasks, median reaction time was not systematically higher in the ASD group. Within the ASD group, there was a moderate positive correlation between reaction times and

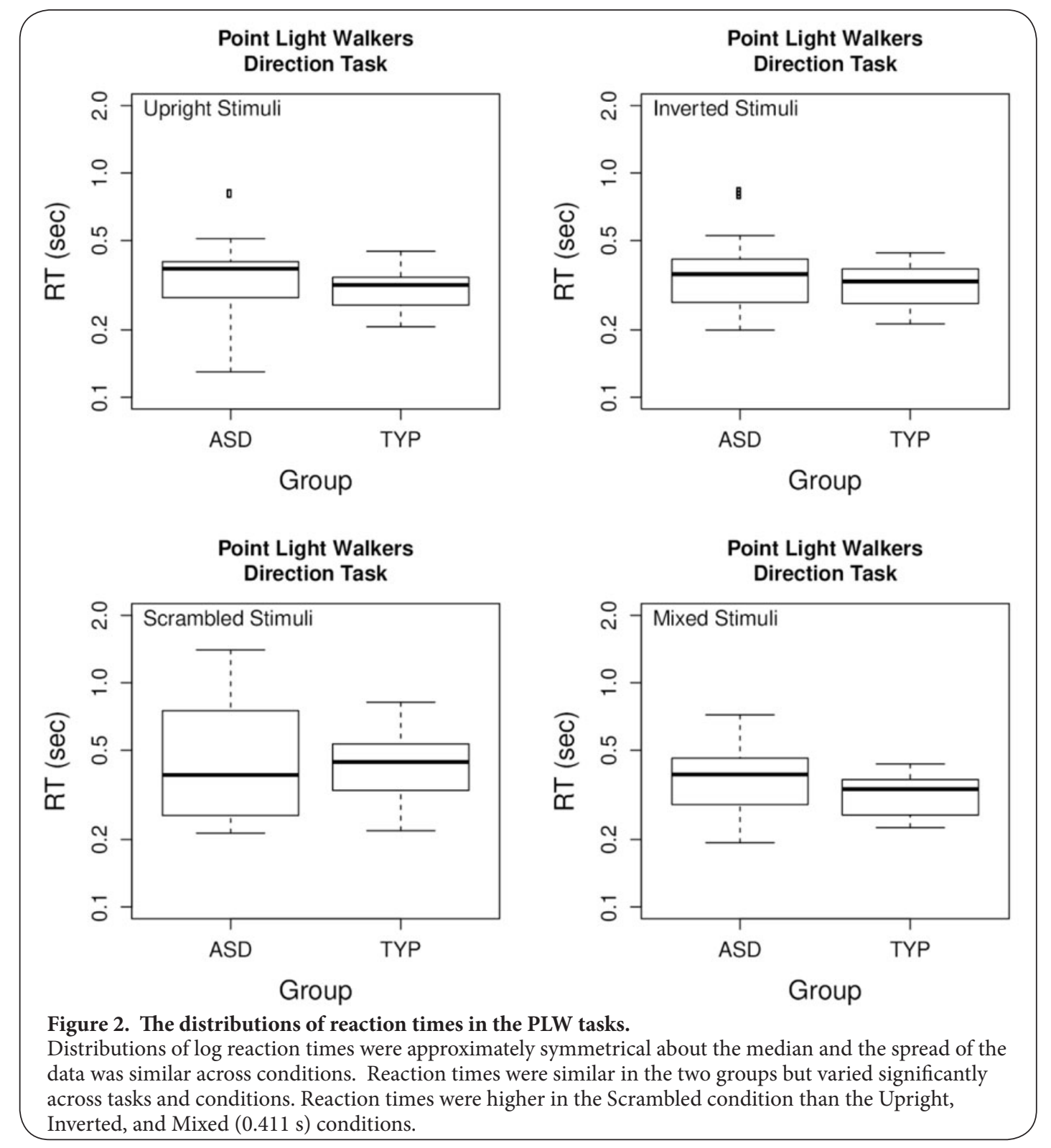


Rutherford et al., Journal of Autism 2020,

Social Affect (SA) ADOS scores that did not reach significance $(r=0.40, p=0.15)$. Log-transformed reaction times were analyzed with a 2 (group: ASD vs Control) x 2 (task: Direction vs. Emotion) x 4 (condition: Upright, Inverted, Scrambled, Mixed) ANOVA. The ANOVA found significant main effects of task $\left(F(1,26)=54.86, \eta_{\mathrm{p}}{ }^{2}=0.68, p<0.001\right)$ and condition $\left(F(1.97,51.10)=13.26, \eta_{\mathrm{p}}{ }^{2}=0.34, p<0.001\right)$. The task $x$ condition interaction $\left(F(1.93,50.23)=3.08, \eta_{p}{ }^{2}=0.11, p=0.06\right)$, the main effect of group $\left(F(1,26)=1.20, \eta_{\mathrm{p}}{ }^{2}=0.04, p=0.28\right)$, and all other effects $\left(F<1, \eta_{\mathrm{p}}{ }^{2}<0.05, p>0.5\right)$ were not significant. The main effect of task reflects the fact that reaction time was significantly greater in the Emotion task ( $M=0.525 \mathrm{~s}$ ) than the Direction task $(M=0.354 \mathrm{~s})$. The main effect of condition was analyzed by conducting pairwise $t$ tests: significant differences were found between mean reaction time in the Scrambled condition and the other three conditions ( $t(13)>3.4, d>0.65$, $p<0.005$ ), and between the Upright and Inverted conditions ( $t$ $(13)=2.41, d=0.45, p=0.023)$. In summary, reaction times were similar in the two groups but varied significantly across tasks and conditions. Specifically, reaction times were on average $171 \mathrm{~ms}$ higher in the Emotion task than the Direction task and were higher in the Scrambled condition ( $M=0.494 \mathrm{~s}$ ) than the Upright ( $M=0.396 \mathrm{~s})$, Inverted $(M=0.429 \mathrm{~s})$, and Mixed (0.411 s) conditions.

\section{Congruency Effects in the Navon Tasks}

Recall that congruency effects were defined as the log difference between reaction times on congruent trials and either incongruent or neutral trials. In the Local task, participants identified the local stimulus elements while trying to ignore the global structure, and therefore a positive congruency effect reflects an inability to ignore the global structure. In the Global task, participants identified the global structure while trying to ignore the local elements, and therefore a positive congruency effect reflects an inability to ignore the local structure. Hence, positive congruency effects in the Local and Global tasks are evidence for Global and Local precedence (or interference), respectively. In the Local task, both groups showed approximately equal congruency effects; however, in the Global task only the autism group exhibited a positive congruency effect. These observations were supported by $t$ tests. Global Precedence in the ASD $(t(13)=5.34, d=1.43$, $p<0.001)$ and Control $(t(13)=8.66, d=2.32, p<0.001)$ groups were significantly different from zero but did not differ from each other $(t(22.7)=0.375, d=0.142, p=0.71)$. However, Local Precedence differed significantly from zero in the ASD group $(t(13)=3.40, d=0.91, p=0.005)$ but not the Control group $(t(13)=0.53, d=0.14, p=0.61)$, and the difference between groups was significant $(t(15.64)=3.08, d=1.16, p=0.007)$.

\section{Global Precedence in the PLW Tasks}

The distributions of the measures of Global Precedence in the PLW tasks are shown in Figure 3. Each measure was based on a comparison of the average performance in the Upright and Mixed conditions and average performance in the Scrambled and Inverted conditions: positive scores indicate that responses were faster or more accurate for Upright/Mixed stimuli than Scrambled/Inverted stimuli. Global Precedence was estimated using response time and accuracy measures in the Emotion task, but (due to ceiling effects on accuracy) only response time in the Direction task. In the Emotion task, Global Precedence measured with reaction time $(M=0.057, t(27)=3.49, d=0.66$, $\mathrm{p}=0.002))$ and accuracy $(\mathrm{M}=0.149, t(27)=6.45, d=1.22, \mathrm{p}<0.001)$ were significantly different from zero; however, in neither case did Global Precedence differ significantly between groups (in both cases, $t(13)<0.7, d<0.25, p>0.5$ ). Similarly, Global
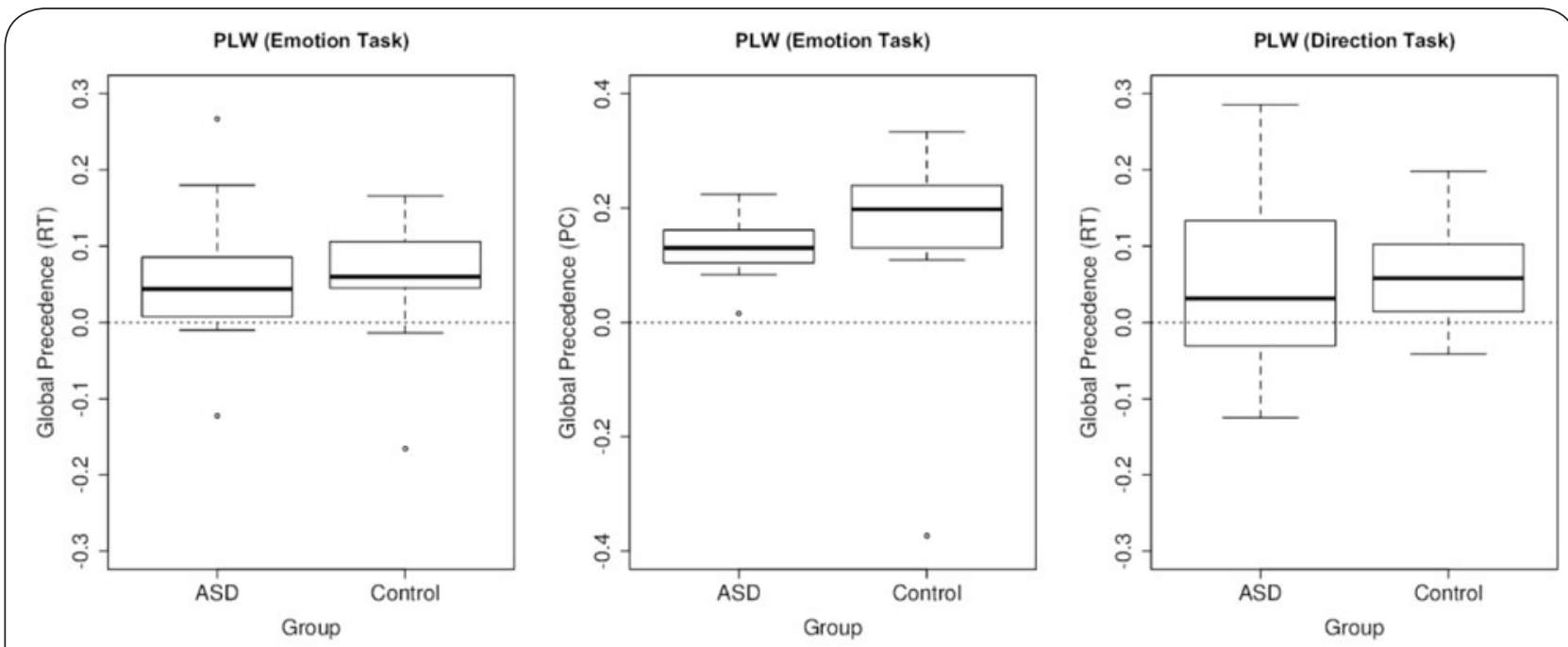

Figure 3. The distributions of the measures of Global Precedence in the PLW tasks.

In the Emotion task, Global Precedence measured with reaction time and response accuracy was significantly different from zero. However, in neither case did Global Precedence differ significantly between groups. 
Rutherford et al., Journal of Autism 2020,

http://www.hoajonline.com/journals/pdf/2054-992X-7-2.pdf

Precedence in the Direction task differed significantly from zero $(M=0.057, t(27)=3.08, d=0.58, p=0.005)$ but did not differ significantly between groups $(t(20.1)=0.216, e=0.08, p=0.83)$.

\section{Are non-social and social Global Precedence measures associated?}

Correlations between the measures of congruency effects in the Navon tasks and Global Precedence in the PLW tasks are presented in Table 2. Our primary hypothesis was that Global Precedence on the Navon task would be correlated with Global Precedence on a social task, specifically the PLW task. After combining data across groups, the correlation between the congruency effect in the local Navon task and the reaction time measure of Global Precedence in the PLWEmotion tasks was significant as assessed by a Spearman's rho analysis $\left(r_{s}(28)=0.47, p=0.006\right)$. The correlation is illustrated as a scatter plot in Figure 4. Follow up Spearman's rho tests indicated that although the correlation was significant in the ASD group $\left(r_{s}(14)=0.48, p=0.02\right)$ it was not significant in the Control group $\left(r_{s}(14)=0.41, p=0.07\right)$. However, contrary to our second prediction, the difference between the two correlations was not significant $(z=0.79, p=0.43)$.

Table 2. Correlations between measures of congruency effects in the Navon tasks and Global Precedence in the PLW tasks. Correlations were calculated after combining data across groups.

\begin{tabular}{llllll}
\hline & $\begin{array}{l}\text { Navon } \\
\text { (Local) }\end{array}$ & $\begin{array}{l}\text { Navon } \\
(\text { Global })\end{array}$ & $\begin{array}{l}\text { PLW } \\
(\text { Emo/RT) }\end{array}$ & $\begin{array}{l}\text { PLW } \\
(\text { Emo/Acc) }\end{array}$ & $\begin{array}{l}\text { PLW } \\
(\text { Dir/RT) }\end{array}$ \\
\hline $\begin{array}{l}\text { Navon } \\
\text { (Local) }\end{array}$ & - & .0057 & $.5186^{*+}$ & .1747 & .2150 \\
\hline $\begin{array}{l}\text { Navon } \\
\text { (Global) }\end{array}$ & .0057 & - & .0023 & -.1808 & .0672 \\
\hline $\begin{array}{l}\text { PLW } \\
\text { (Emo/RT) }\end{array}$ & $.5186^{*+}$ & .0023 & - & $.4609^{*}$ & $.4483^{*}$ \\
\hline $\begin{array}{l}\text { PLW } \\
\text { (Emo/Acc) }\end{array}$ & .1747 & -.1808 & $.4609^{*}$ & - & -.2182 \\
\hline PLW (Dir/RT) & .2150 & .0672 & $.4483^{*}$ & -.2182 & - \\
\hline
\end{tabular}

${ }^{*} \mathrm{p}<0.05$ (uncorrected); $+\mathrm{p}<0.05$ (corrected)

\section{Discussion}

A primary goal of this study was to test whether Global Precedence, as evidenced by global interference on the identification of local elements in the Navon task, correlated with our index of Global Precedence that was based on performance on the Emotion trials of the point-light walker task. Consistent with the predictions of the Weak Central Coherence theory, we found a statistically significant positive correlation in the autism group but not in the control group. The correlation between Global Precedence on the Navon task and Global Precedence on the point-light walker task is consistent with the hypothesis that there is a relationship between weak central coherence and social perception in autism. There was not a significant between-group difference in the magnitude of this correlation.

This study was designed to examine the relationship be-

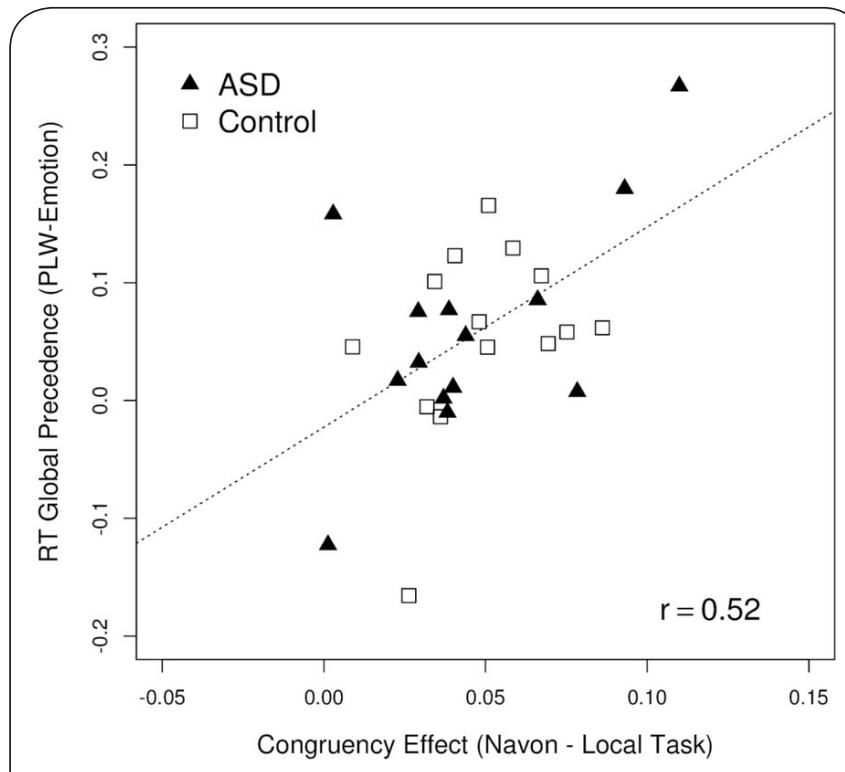

Figure 4. A scatter plot illustrating the association between the congruency effect in the Local Navon task and the reaction time measure of Global Precedence in the PLW-Emotion task. The dashed line is the regression line. The overall correlation $(\mathrm{r}=0.52)$ was statistically significant, but there is little evidence that the association differed between groups.

tween perceptual tendencies towards local processing, or Weak Central Coherence, on a social and a non-social task. More broadly, it addresses the question of why both Weak Central Coherence and difficulties in social processing coexist in populations with autism, when the two do not seem to rely on obviously similar psychological processing. It is typical in the field of autism research to deal with non-social cognitive anomalies [19] separately from social perception $[12,68]$ as if they were psychologically distinct, with some authors explicitly claiming distinct processing $[\mathbf{1 4 , 2 0}]$; but see 4$)$. Few previous studies have examined the relationship between global processing and performance on social tasks, but [63] reported a strong correlation between attention to detail and social problems measured by the Social Responsiveness Scale among young people with an autism diagnosis. This association is potentially relevant to the daily lives of some individuals, as social deficits are some of the most clinically noticeable in ASD. The current study suggests that there may be a psychological association between the two task types; the two characteristics of autism may not be as separate as is commonly assumed.

The current finding is consistent with recent work involving fMRI measurements in adolescents on the autism spectrum and controls. Participants viewed social stimuli that were depicted using geometric shapes. The task either required them to identify the emotion portrayed or indicate whether a specific shape was present. The ASD group showed relatively less connectivity between body perception and motor 
Rutherford et al., Journal of Autism 2020,

networks, suggesting a possible difference with respect to mirroring. The authors concluded that social cognitive factors, not local processing, underlies the observed between-group differences in performance [25].

In the current study, the analysis of performance on the Navon task also provides support for the Weak Central Coherence theory with respect to non-social perception. Neurotypical individuals show a global bias on this task $[3,41]$. The weak central coherence theory would predict that this tendency would be absent or relatively weak in the autism spectrum group. On the Navon task, we found significant evidence of a group difference: reaction times on correct trials were longer in participants on the spectrum than Control participants, and this group difference was greater for the Global task than the Local task. This reflects more facile processing of global information (over local) on the part of neurotypical observers only. This finding agrees with a recent meta-analysis that reported slower performance on global processing among those on the autism spectrum, especially when local interference is present. The authors of this meta-analysis concluded that this phenomenon is best characterized as local to global interference among those on the autism spectrum rather than global to local inference in the typically developing control group $[57,64]$.

Note that our analyses rely on measures of reaction time, and that we did not find group differences in accuracy on either our social or non-social measures. This is consistent with the way global and local processing differences are characterized in the recent literature: A recent meta-analysis reports measurable group differences in global processing when the dependent variable is reaction time (Hedges' $g=$ 0.43 ) but not when the dependent variable is accuracy [64]. In addition, this same meta-analysis reported that the greatest measured group difference occurs when the task involves hierarchical letters or figures [64], which is true of both the Navon stimuli and the scrambled point-light walker used in the current study.

Group differences in cognitive function cannot account for our results. First, IQ below 70 was used as an exclusion criterion. Second, the two groups were matched on IQ. Third, our conclusions are not based on group differences in performance, but rather on the relationship between a tendency toward Global Precedence across tasks. We report a nominally stronger correlation $(r=0.65)$ between the two Global Precedence measures in the autism group compared to the relationship in the control group $(r=0.39)$ but note that this difference was not statistically significant.

We designed this experiment to test whether one's tendency towards global processing might be a candidate for explaining differences in social perception as predicted by the classic formulation of Weak Central Coherence theory, but note that the Enhanced Perceptual Functioning (EPF) account of autistic perceptual processing would also predict this outcome $[37,38]$. The Enhanced Perceptual Functioning account is broader than Weak Central Coherence theory. It encapsulates everything that the Weak Central Coherence theory does (particularly in its Principle 1 which describes locally oriented visual and auditory perception and enhanced low-level information processing), but also accounts for some findings that the Weak Central Coherence theory cannot. The EPF frames what we call local bias as a default setting, whereby autistic perception is more locally oriented than neurotypical perception. It further posits that higher-order processing, what we call global processing, is optional in autism and mandatory in neurotypical observers. It also allows that people with autism may update this strategy depending upon the demands of the current task. Our experiment was not designed to contrast these two accounts; rather, our results are consistent with both.

In contrast to the WCC perspective, the EPF describes taskdependent flexibility with respect to whether an individual with autism shows a local bias. This is consistent with the findings of Plaisted and colleagues [47] who found that children with autism showed the expected local processing tendency, but it also described flexibility in processing. When asked explicitly to process at the global level, autistic participants' patterns of performance match those of neurotypicals. They did not find any such flexibility in their neurotypical control group, only the expected global processing bias [47]. The current study was not designed to manipulate local versus global processing, only to measure the extent to which participants showed a global bias. Our findings, therefore, should be taken as an indication of what the EPF perspective would call default settings, leaving the question of flexibility open.

It would be incorrect to think of higher (or lower) correlations between our two measures of Global Precedence as worse performance. Central coherence is not a skill that might be impaired, but as a style of perception. A better check on performance would be accuracy, for which we found no group differences with respect to the Navon task or the Point Light Walker task.

Finally, it would be best to understand these results as preliminary. First, this is the first study that directly tests the relationship between Global Precedence and social perception. Second, although there was a significant correlation between these two constructs in the group with autism spectrum, the difference between correlations across the two groups was not significant. Third, this study tested 14 participants in each group, which is a relatively small sample for a behavioral study. Lastly, participants with an ASD diagnosis in this study were relatively high functioning young adults, and it is impossible to know whether these results would generalize to a lower-functioning group with ASD. That said, global integration has been reported to be associated with IQ for participants with ASD but not controls [8], so we would not expect a lower-functioning sample to behave more like typical controls than the current sample did.

To summarize, overall, we provide support for the idea 
Rutherford et al., Journal of Autism 2020,

that weak central coherence is related to social perception in autism. The relationship between global processing across the social and non-social conditions was significant for the group on the autism spectrum and was positive though non-significant for the control group. This relationship did not differ significantly between groups. Our results suggest that there may be global processing, or central coherence, that underpins performance on both tasks.

\section{Competing interests}

The authors declare that they have no competing interests.

\section{Authors' contributions}

\begin{tabular}{|l|c|c|c|c|}
\hline Authors' contributions & MDR & NT & PJB & ABS \\
\hline Research concept and design & $\checkmark$ & $\checkmark$ & $\checkmark$ & $\checkmark$ \\
\hline Collection and/or assembly of data & -- & $\checkmark$ & -- & -- \\
\hline Data analysis and interpretation & $\checkmark$ & -- & $\checkmark$ & $\checkmark$ \\
\hline Writing the article & $\checkmark$ & -- & -- & -- \\
\hline Critical revision of the article & $\checkmark$ & -- & $\checkmark$ & $\checkmark$ \\
\hline Final approval of article & $\checkmark$ & -- & -- & -- \\
\hline Statistical analysis & -- & -- & $\checkmark$ & -- \\
\hline
\end{tabular}

\section{Acknowledgement}

This study was supported by a Discovery Grant given to M.D. Rutherford from the Natural Sciences and Engineering Research Council of Canada, NSERC.

\section{Publication history}

Editor: David Reiss, Imperial College London, UK. Received: 22-Aug-2020 Final Revised: 29-Sept-2020 Accepted: 08-Oct-2020 Published: 15-Oct-2020

\section{References}

1. Almeida, R. A., Dickinson, J. E., Maybery, M. T., Badcock, J. C., \& Badcock, D. R. (2014). Enhanced global integration of closed contours in individuals with high levels of autistic-like traits. Vision Research, 103, 109-115. doi:10.1016/j.visres.2014.08. 015.

2. Atkinson, A. P. (2009). Impaired recognition of emotions from body movements is associated with elevated motion coherence thresholds in autism spectrum disorders. Neuropsychologia, 47, 3023-3029.

3. Badcock, J. C., Whitworth, F. A., Badcock, D. R., \& Lovegrove, W. J. (1990). Low-frequency filtering and the processing of local-global stimuli. Perception 19(5), 617-629.

4. Baron-Cohen, S., \& Swettenham, J. (1997). Theory of mind in autism: Its relationship to executive function and central coherence. Handbook of Autism and Pervasive Developmental Disorders, 880-893.

5. Behrmann, M., Thomas, C., \& Humphreys, K. (2006). Seeing it differently: visual processing in autism. Trends in cognitive sciences, 10(6), 258-264.

6. Bertschy, K., Skorich, D. P., \& Haslam, S. A. (2019). Self-categorization and Autism: Exploring the Relationship Between Autistic Traits and Ingroup Favouritism in the Minimal Group Paradigm. Journal of Autism and Developmental Disorders, 1-16.

7. Blake, R., Turner, L. M., Smoski, M. J., Pozdol, S. L., \& Stone, W. L. (2003). Visual recognition of biological motion is impaired in children with autism. Psychological Science, 14, 151-157.

8. Booth, R. D., \& Happé, F. G. (2018). Evidence of reduced global processing in autism spectrum disorder. Journal of autism and developmental disorders, 48(4), 1397-1408.

9. Brainard, D. H. (1997). The Psychophysics Toolbox. Spatial Vision, 10, 433-436.

10. Cutting, J. E. (1978). A program to generate synthetic walkers as dynamic point light displays. Behaviour Research Methods and Instrumentation, 10, 91-94.

11. Constantino, J. N., Davis, S. A., Todd, R. D., Schindler, M. K., Gross, M. M., Brophy, S. L., ... \& Reich, W. (2003). Validation of a brief quantitative measure of autistic traits: comparison of the social responsiveness scale with the autism diagnostic interview-revised. Journal of autism and developmental disorders, 33(4), 427-433.

12. Elsabbagh, M., \& Johnson, M. H. (2016). Autism and the social brain: The first-year puzzle. Biological Psychiatry, 80(2), 94-99.

13. Frith, U. (1989). Autism: Explaining the Enigma. Oxford: Blackwell Scientific Publications.

14. Frith, U. \& Happé, F. (1994). Autism: Beyond "theory of mind". Cognition, 50, 115-132.

15. Grelotti, D. J., Gauthier, I., \& Schultz, R. T. (2002). Social interest and the development of cortical face specialization: What autism teaches us about face processing. Developmental Psychobiology, 40, 213-225.

16. Grezes, J., Fonlupt, P., Bertenthal, B., Delon-Martin, C., Segebarth, C., \& Decety, J. (2001). Does perception of biological motion rely on specific brain regions?. Neuroimage, 13(5), 775-785.

17. Grossman, E. D. (2006). in Perception of Biological Motion. Human body perception from the inside out, 361.

18. Hadad, B.-S., \& Ziv, Y. (2015). Strong bias towards analytic perception in ASD does not necessarily come at the price of impaired integration skills. Journal of Autism and Developmental Disorders, 45(6), 1499-1512. doi:10.1007/s10803-014-2293-5.

19. Happé, F. G. (1996). Studying weak central coherence at low levels: Children with autism do not succumb to visual illusions. A research note. Journal of Child Psychology and Psychiatry, 37(7), 873-877

20. Happé, F. G. (1997). Central coherence and theory of mind in autism: Reading homographs in context. British Journal of Developmental Psychology, 15(1), 1-12

21. Happé, F. (1999). Autism: cognitive deficit or cognitive style? Trends in Cognitive Sciences, 3, 216-222.

22. Happé, F. G., \& Booth, R. D. (2008). The power of the positive: Revisiting weak coherence in autism spectrum disorders. Quarterly journal of experimental psychology, 61(1), 50-63.

23. Happé, F. \& Frith, U. (2006). The Weak Central Coherence Account: Detail-focussed Cognitive Style in Autism Spectrum Disorders. Journal of Autism and Developmental Disorders, 36, 5-25.

24. Happé, F. G. E. (1996). Studying weak central coherence at low levels: Children with autism do not succumb to visual illusions. Journal of Child Psychology and Psychiatry, 37, 873-877.

25. Herringshaw, A. J., Kumar, S. L., Rody, K. N., \& Kana, R. K. (2018). Neural correlates of social perception in children with autism: Local versus global preferences. Neuroscience, 395, 49-59

26. Hubert, B., Wicker, B., Moore, D. G., Monfardini, E., Duverger, H., Da Fonseca, D. et al. (2006). Brief Report: Recognition of emotional and non-emotional biological motion in individuals with autistic spectrum disorders. Journal of Autism and Developmental Disorders, 37, 13861392.

27. Hulley SB, Cummings SR, Browner WS, Grady D, Newman TB. Designing clinical research : an epidemiologic approach. 4th ed. Philadelphia, PA: Lippincott Williams \& Wilkins; 2013.

28. Jolliffe, T. \& Baron-Cohen, S. (1997). Are people with autism and Asperger syndrome faster than normal on the Embedded Figures Test? Journal of Child Psychology and Psychiatry, 38, 527-534.

29. Joseph, R. M. \& Tanaka, J. (2002). Holistic and part-based face recognition in children with autism. Journal of Child Psychology and Psychiatry, 43, 1-14.

30. Kimchi, R. (1992). Primacy of wholistic processing and global/loca paradigm: a critical review. Psychological bulletin, 112(1), 24.

31. Koldewyn, K., Whitney, D., \& Rivera, S. M. (2011). Neural correlates of coherent and biological motion perception in autism. Developmental Science, 14(5), 1075-1088.

32. Langdell, T. (1978). Recognition of Faces - Approach to Study of Autism. Journal of Child Psychology and Psychiatry and Allied Disciplines, 19 
Rutherford et al., Journal of Autism 2020,

255-268

33. Lord, C., Risi, S., Lambrecht, L., Cook, E. H., Leventhal, B. L., DiLavore, P. C. et al. (2000). The Autism Diagnostic Observation Schedule-Generic: A standard measure of social and communication deficits associated with the spectrum of autism. Journal of Autism and Developmental Disorders, $30,205-223$.

34. Lord, C., Rutter, M., \& LeCouteur, A. (1994). Autism Diagnostic Interview-Revised: A revised version of a diagnostic interview for caregivers of individuals with possible pervasive developmental disorders. Journal of Autism and Developmental Disorders, 24, 659-685.

35. Maxwell, S. E., \& Delaney, H. D. (2004). Introduction to Model Comparisons: One-Way Between-Subjects Designs. Designing experiments and analyzing data: A model of comparison perspective.

36. Milne, E., \& Szczerbinski, M. (2009). Global and local perceptual style, field-independence, and central coherence: An attempt at concept validation. Advances in Cognitive psychology, 5, 1.

37. Mottron, L., \& Burack, J. (2001). Enhanced perceptual functioning in the development of persons with autism. The Development of Autism Perspectives from Theory and Research, 131-148.

38. Mottron, Laurent, Dawson, M., Soulieres, I., Hubert, B., \& Burack, J. (2006). Enhanced perceptual functioning in autism: An update, and eight principles of autistic perception. Journal of Autism and Developmental Disorders, 36(1), 27-43.

39. Nagai, M., Bennett, P. J., Rutherford, M. D., Gaspar, C. M., Kumada, T., \& Sekuler, A. B. (2013). Comparing face processing strategies between typically-developed observers and observers with autism using subsampled-pixels presentation in response classification technique. Vision research, 79, 27-35.

40. Navon, D. (1977). Forest before trees: The precedence of global features in visual perception. Cognitive Psychology, 9, 353-383.

41. Navon, D. (1981). Do attention and decision follow perception? Journal of Experimental Psychology: Human Perception and Performance, 7, 1175-1182.

42. O'Hearn, K., Franconeri, S., Wright, C., Minshew, N., \& Luna, B. (2013). The development of individuation in autism. Journal of Experimental Psychology: Human Perception and Performance, 39(2), 494.

43. O’Riordan, M. A., Plaisted, K. C., Driver, J., \& Baron-Cohen, S. (2001). Superior visual search in autism. Journal of Experimental Psychology: Human Perception and Performance, 27, 719-730.

44. Parron, C., Da Fonseca, D., Santos, A., Moore, D. G., Monfardini, E., \& Deruelle, C. (2008). Recognition of biological motion in children with autistic spectrum disorders. Autism, 12, 261-274.

45. Pelli, D. G. (1997). The VideoToolbox software for visual psychophysics: Transforming numbers into movies. Spatial Vision, 10, 437-442.

46. Plaisted, K., O'Riordan, M., \& Baron-Cohen, S. (1998). Enhanced Visual Search for a Conjunctive Target in Autism: A Research Note. Journal of Child Psychology and Psychiatry and Allied Disciplines, 39, 777-783.

47. Plaisted, K., Swettenham, J., \& Rees, L. (1999). Children with autism show local precedence in a divided attention task and global precedence in a selective attention task. The Journal of Child Psychology and Psychiatry and Allied Disciplines, 40(5), 733-742.

48. R Core Team (2018) R: A Language and Environment for Statistical Computing. R Foundation for Statistical Computing, Vienna.

49. Roether, C. L., Omlor, L., Christensen, A., \& Giese, M. A. (2009). Critical features for the perception of emotion from gait. Journal of Vision, 9 , 1-32.

50. Rutherford, M. D., Clements, K. A., \& Sekuler, A. B. (2007). Differences in discrimination of eye and mouth displacement in autism spectrum disorders. Vision research, 47(15), 2099-2110.

51. Rutherford, M. D. \& McIntosh, D. N. (2007). Rules versus Prototype Matching: Strategies of Perception of Emotional Facial Expressions in the Autism Spectrum. Journal of Autism and Developmental Disorders, 37 187-196.

52. Rutherford, M. D., Pennington, B. F., \& Rogers, S. J. (2006). The Perception of Animacy in Young Children with Autism. Journal of Autism and Developmental Disorders, 36, 893-992.

53. Rutherford, M. D., Troubridge, E., \& Walsh, J. (2012). Visual afterimages of emotional faces in high functioning autism. Journal of Autism and Developmental Disorders, 42, 221-229.

54. Salter, G., Seigal, A., Claxton, M., Lawrence, K., \& Skuse, D. (2008). Can autistic children read the mind of an animated triangle? Autism, 12, 349-371.

55. Shah, A. \& Frith, U. (1983). An Islet of Ability in Autistic-Children - A Research Note. Journal of Child Psychology and Psychiatry and Allied Disciplines, 24, 613-620.

56. Shah, A. \& Frith, U. (1993). Why do autistic individuals show superior performance on the block design task? Journal of Child Psychology and Psychiatry, 34, 1351-1364.

57. Simmons, D. R., Robertson, A. E., McKay, L. S., Toal, E., McAleer, P., \& Pollick, F. E. (2009). Vision in autism spectrum disorders. Vision research, 49(22), 2705-2739.

58. Skorich, D. P., Gash, T. B., Stalker, K. L., Zheng, L., \& Haslam, S. A. (2017). Exploring the cognitive foundations of the shared attention mechanism: Evidence for a relationship between self-categorization and shared attention across the autism spectrum. Journal of Autism and Developmental Disorders, 47(5), 1341-1353.

59. Skorich, D. P., May, A. R., Talipski, L. A., Hall, M. H., Dolstra, A. J., Gash, T. B., \& Gunningham, B. H. (2016). Is social categorization the missing link between weak central coherence and mental state inference abilities in autism? Preliminary evidence from a general population sample. Journal of Autism and Developmental Disorders, 46(3), 862-881.

60. Stevenson, R. A., Sun, S. Z., Hazlett, N., Cant, J. S., Barense, M. D., \& Ferber, S. (2018). Seeing the forest and the trees: default local processing in individuals with high autistic traits does not come at the expense of global attention. Journal of autism and developmental disorders, 48(4), 1382-1396.

61. Troje, N. F., \& Aust, U. (2013). What do you mean with "direction"? Local and global cues to biological motion perception in pigeons. Vision Research, 79, 47-55.

62. Troje, N. F., \& Chang, D. H. (2013). Shape-independent processing of biological motion. People watching: Social, perceptual, and neurophysiological studies of body perception, 82-100.

63. Van Eylen, L., Boets, B., Steyaert, J., Wagemans, J., \& Noens, I. (2018). Local and global visual processing in autism spectrum disorders: Influence of task and sample characteristics and relation to symptom severity. Journal of Autism and Developmental Disorders, 48(4), 13591381.

64. Van der Hallen, R., Evers, K., Brewaeys, K., Van den Noortgate, W., \& Wagemans, J. (2015). Global processing takes time: A meta-analysis on local-global visual processing in ASD. Psychological Bulletin, 141(3), 549.

65. Wang, L., Mottron, L., Peng, D., Berthiaume, C., \& Dawson, M. (2007). Local bias and local-to-global interference without global deficit: A robust finding in autism under various conditions of attention, exposure time, and visual angle. Cognitive Neuropsychology, 24(5), 550-574. doi:10.1080/ 13546800701417096.

66. Wechsler, D. (2008). Wechsler Adult Intelligence Scale-Fourth Edition (WAIS-IV). San Antonio, TX: The Psychological Corporation.

67. Wertheimer, M. (1938). Laws of organization in perceptual forms. In W.Ellis (Ed.), A source book of Gestalt psychology (pp. 71-88). London: Routledge.

68. Yang, D. Y.-J., Rosenblau, G., Keifer, C., \& Pelphrey, K. A. (2015). An integrative neural model of social perception, action observation, and theory of mind. Neuroscience \& Biobehavioral Reviews, 51, 263-275.

69. Young, A. W., Hellawell, D., \& Hay, D. C. (1987). Configurational information in face perception. Perception, 16, 747-759.

\section{Citation:}

Rutherford MD, Trivedi N, Bennett PJ and Sekuler AB. Weak Central Coherence Contributes to Social Perceptual Deficits in autism. J Autism. 2020; 7:2. http://dx.doi.org/10.7243/2054-992X-7-2 